\title{
Magnetic brightening of dark excitons and its dynamics in monolayer $\mathrm{WSe}_{2}$
}

Railson Vasconcelos*1(PG), Helena Bragança ${ }^{1}(\mathrm{PG}), \mathrm{Qu}$ Fanyao $^{1}(\mathrm{PQ})$. railson.c.vasconcelos@gmail.com

${ }^{1}$ Instituto de Física, Universidade de Brasília, Brasília-DF 70919-970, Brazil.

Keywords: Dark excitons, monolayer, magnetic field.

\section{Introduction}

Monolayers of semiconducting transition metal dichalcogenides (TMDCs) such as $\mathrm{WSe}_{2}$ have drawn considerable attention [1] following the discovery of direct band gap at the two inequivalent $\mathrm{K}$ and $\mathrm{K}$ ' valleys. Owing to the broken inversion symmetry, it allows for optical generation and manipulation of the valley polarization.

Because of the two-dimensional nature of the TMDCs, out-of-plane and in-plane magnetic fields present distinct impacts on the valley physics. The former breaks time reversal symmetry and lifts valley degeneracy due to Zeeman effect. The in-plane magnetic field induces a mixing of different spin states, i.e., bright and dark states. Thus, it enables dark exciton brightening [2,3], which allows us to explore the dynamics and valley properties of these states with lifetimes orders-of-magnitude longer than that of the bright states.

In this work, we aim to establish the full picture of dark exciton brightening and dark exciton engaged valley dynamics in $\mathrm{WSe}_{2}$ subjected magnetic field. We focus on the dark exciton valley dynamics to demonstrate that not only the magnitude but also the angle of magnetic field can be used as tools to achieve desired PL intensity and high valley polarization.

\section{Methodology}

Below, we present our theory to describe bright and dark excitons in monolayer $\mathrm{WSe}_{2}$ subjected to magnetic field. We start from the description of the exciton Hamiltonian, then we construct a set of rate equations to describe the dark exciton engaged valley dynamics.

The general matrix for the A exciton under a tilted field written in the exciton basis is:

$$
\hat{H}_{x}^{\tau A}=\left(\begin{array}{cc}
E_{b}^{A \tau}\left(B_{\perp}\right) & \frac{g_{e} \mu_{B}}{2} B_{-} \\
\frac{g_{e} \mu_{B}}{2} B_{+} & E_{d}^{A \tau}\left(B_{\perp}\right)
\end{array}\right)
$$

Then, we obtain the set of rate equations describing the valley dynamics for the mixed bright-dark states.

$$
\begin{aligned}
& \frac{d n_{b}^{\text {mix }}}{d t}=g-\frac{n_{b}^{\text {mix }}}{\bar{\tau}_{r b}}-\frac{n_{b}^{\text {mix }}}{\bar{\tau}_{s k x}}-\frac{n_{b}^{\text {mix }}}{\tau_{b d}}+\frac{n_{b}^{\text {mix }^{\prime}}}{\bar{\tau}_{s k x}}+\frac{n_{d}^{\text {mix }}}{\tau_{b d}} u \\
& \frac{d n_{d}^{\text {mix }}}{d t}=-\frac{n_{d}^{\text {mix }}}{\bar{\tau}_{r d}}-\frac{n_{d}^{\text {mix }}}{\tau_{b d}} u-\frac{n_{d}^{\text {mix }}}{\tilde{\tau}_{s k x}}+\frac{n_{b}^{\text {mix }}}{\tau_{b d}}+\frac{n_{d}^{\text {mix }^{\prime}}}{\tilde{\tau}_{s k x}} \\
& \frac{d n_{b}^{\text {mix }^{\prime}}}{d t}=g^{\prime}-\frac{n_{b}^{\text {mix }^{\prime}}}{\bar{\tau}_{r b}}-\frac{n_{b}^{\text {mix }^{\prime}}}{\bar{\tau}_{s k x}}-\frac{n_{b}^{\text {mix }^{\prime}}}{\tau_{b d}}+\frac{n_{b}^{\text {mix }}}{\bar{\tau}_{s k x}}+\frac{n_{d}^{\text {mix }^{\prime}}}{\tau_{b d}} u \\
& \frac{d n_{d}^{\text {mix }^{\prime}}}{d t}=-\frac{n_{d}^{\text {mix }^{\prime}}}{\bar{\tau}_{r d}}-\frac{n_{d}^{\text {mix }^{\prime}}}{\tau_{b d}} u-\frac{n_{d}^{\text {mix }^{\prime}}}{\tilde{\tau}_{s k x}}+\frac{n_{b}^{\text {mix }^{\prime}}}{\tau_{b d}}+\frac{n_{d}^{\text {mix }}}{\tilde{\tau}_{s k x}},
\end{aligned}
$$


We compute the steady-state PL intensity by solving the coupled rate equations with the left-hand sides equal to zero $(\mathrm{dn} / \mathrm{dt}=0)$, and further determine the valley polarization.

\section{Results}

In Fig. 1 we show our results for the influence of an external magnetic field to the monolayer on the excitonic optical response of $\mathrm{WSe}_{2}$.
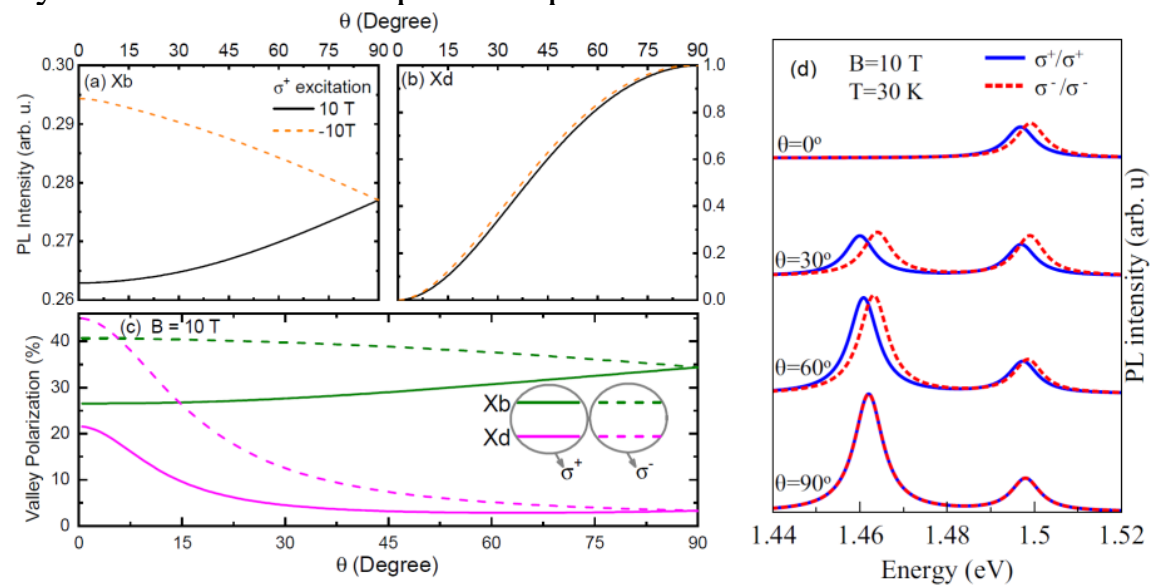

Fig. 1. PL intensities at $30 \mathrm{~K}$ of bright (a) and dark (b) excitons in the $\mathrm{K}$ valley of $\mathrm{WSe}_{2}$ under $\sigma+$ excitation as a function of the tilt angle of magnetic fields for $\mathrm{B}=-10$ (red) and $+10 \mathrm{~T}$ (black). (c) Valley polarization of bright (green) and dark (magenta) excitons as a function of tilt angle for $\mathrm{B}=10 \mathrm{~T}$. In (c), solid and dashed lines correspond to excitation $\sigma^{+}$and $\sigma^{-}$, respectively. (d) Excitonic PL spectra under a magnetic field of $10 \mathrm{~T}$, for tilt angle $\theta$. Solid blue and dashed red lines correspond to and co-polarization excitation.

\section{Conclusions}

We have investigated the impact of tilt magnetic field with an arbitrary tilt angle on dark exciton engaged valley dynamics and valley polarization. For out-of plane field, we find that the photoluminescence (PL) intensity, changes with B monotonically, but exhibits opposite trends under optical excitations with different polarization helicities, as a result of broken valley degeneracy. For in-plane field, which mixes bright and dark states and further enables dark exciton brightening, we find that the PL intensity and valley polarization of the "brightened" dark exciton, having a parabolic dependence on B field, is independent of pumping polarization helicity.

\section{Acknowledgements}

This work was supported by CNPq, CAPES, FAPDF, FAPESP, and the Natural Science Foundation of China (Grant No. 11004120).

\section{References}

1. A. M. Jones et al., Nat Nano 8, 634 (2013).

2. M. R. Molas, C. Faugeras, A. O. Slobodeniuk, K. Nogajewski, M. Bartos, D. M. Basko, and M. Potemski, 2D Mater. 4, 021003 (2017).

3. X. X. Zhang, T. Cao, Z. G. Lu, Y. C. Lin, F. Zhang, Y.Wang, Z. Q. Li, J. C. Hone, J. A. Robinson, D. Smirnov, S. G. Louie, and T. F. Heinz, Nat. Nanotechnol. 12, 883 (2017). 